\title{
Promising biomarkers in pediatric chronic kidney disease through the kaleidoscope of CAKUT background complexity
}

\author{
Aleksandra Stankovic ${ }^{1}$ (D) \\ Received: 28 October 2020 / Accepted: 26 November 2020 / Published online: 6 January 2021 \\ (C) IPNA 2021
}

\section{Introduction}

Chronic kidney disease (CKD) causes and progression in children may significantly differ from those in adults, and efforts have been made in recent years to improve understanding and elucidation of risk factors, disease course, and treatment for renal protection in pediatric patients. It is known that congenital anomalies of the kidney and urinary tract (CAKUT) are the primary cause of CKD and the prevalence of congenital hydronephrosis increases each year at pan-European level, reaching a $2.6 \%$ (95\% CI: $1.7 \% ; 3.5 \%)$ increase in 2017 in comparison to 2008 (average of 11 per 10,000 births) [1]. In spite of frequent prenatal ultrasound screening and successful surgery after birth, some proportion of children will continue to have persistent kidney damage. Studies estimating CKD that involve only children with CAKUT are scarce, and this makes the identification of robust and reproducible biomarkers of disease outcome prediction difficult. A recent article sheds new light on this issue and proposes a possible urine biomarker, which is predictive for the progressive deterioration of kidney function, by monitoring fifty children aged $<14$ years for 12 months who were diagnosed with congenital uropathies: pelviureteric junction obstruction (PUJO), posterior urethral valve (PUV), and vesicoureteral reflux (VUR) [2].

\section{CAKUT pathogenesis and management as underlying factor for CKD}

CAKUT includes a variety of anatomical and histological malformations implying both obstructive and functional

Aleksandra Stankovic

alexas@vinca.rs

1 Vinca Institute of Nuclear Sciences-National Institute of the Republic of Serbia, University of Belgrade, Department of Health and

Environment, Laboratory for Radiobiology and Molecular Genetics, University of Belgrade, Mike Petrovica Alasa 12/14, P.O. Box 522, Belgrade 11001, Serbia abnormalities. Most CAKUT patients are diagnosed antenatally, yet many remain undiagnosed until manifestations of symptoms after birth followed by urinary tract infections. The complex etiology of CAKUT, along with its multifactorial nature and postnatal disease management, results in uneven progression to CKD. Pathogenesis of progressive kidney disease starts during fetal life and includes a spectrum of pathological changes such as outflow abnormalities (PUJO, PUV, VUR, megaureter and duplex collecting system) and kidney agenesis, dysplasia, hypoplasia, decrease in kidney size and nephron number, tubulointerstitial changes, and collecting duct epithelium remodeling. Variability in severity and duration of these changes often induces variability in subsequent kidney damage, in spite of antenatal diagnosis of CAKUT and surgical management of obstructions, which could be followed by both proteinuria and hypertension and, in the long-term, CKD.

Only a few studies on the epidemiology of CKD in children are available, especially for children with CAKUT [3]. The CKiD study for prospective observation of CKD onset in children recruited more than 1000 children from 48 North American pediatric nephrology centers over 5 years, strengthening the longitudinal approach to overcome under-diagnosed and underreported CKD in children. Nevertheless, the estimation of CKD biomarkers in congenital obstructive uropathy in CKiD was performed in 22 cases (receiving kidney replacement therapy) and 22 controls, aged 9-13 years at the baseline. The study of Anand et al. adds value to previous research by 1 -year follow-up of 50 consecutive children with congenital uropathies, aged $<14$ years, and 25 matched population controls in India [2]. Just a short description of the designs of recent studies indicates existing variations among them and the need for a more unified approach that could become part of CKD prevention strategies worldwide, including biomarker assessment. However, currently there is large variation in the methods used and approaches taken by different countries regarding CKD, and the findings have shown poor reproducibility, as reviewed in [4]. 
Different classification systems for CKD severity required a unified approach, independent of cause and applicable to children, which was begun in 2002 [5], and consequently new pediatric registries have started to appear. Still, the methodology for estimation of GFR has limitations in application to children under 2 years of age, especially in the early phases of kidney injury [6]. CKD is currently defined by a presence of kidney damage (e.g., any structural or functional abnormality involving pathological, laboratory, or imaging findings) for $\geq 3$ months or a GFR $<60 \mathrm{ml} / \mathrm{min} /$ $1.73 \mathrm{~m}^{2}$ for $\geq 3$ months. In the study of Anand et al., $53 \%$ of the children had a GFR $<60 \mathrm{ml} / \mathrm{min} / 1.73 \mathrm{~m}^{2}$ comprising the group in which the authors aimed to determine urinary biomarkers of progressive deterioration of kidney function [2]. The majority of children who belonged to the progressive group (either by GFR value or new kidney scars on DMSA) had PUV as the primary diagnosis [2].

\section{Urinary biomarkers of CKD in CAKUT}

Urine is a useful source of biomarker discovery and monitoring of function during physiological processes and disease. It is available and assumes noninvasive collection methods of biological material that contains cells, nucleic acids, and proteins within the urinary tract yet requires standardization of collection, handling, and storage of samples towards development of reliable and reproducible biomarkers [7].

Studies dealing with urinary biomarkers as indicators of kidney damage in pediatric populations with CAKUT are scarce and often do not include GFR estimation $[8,9]$. The study of Anand and colleagues proposes a novel urine/serum putative biomarker for CKD in CAKUT, including prospective measurements of GFR during 1-year follow-up [2]. The authors measured TFF1, TFF3, NGAL, and microalbuminuria and revealed that although each of these biomarkers had an area under the curve $(\mathrm{AUC})>0.83$, the elevation of TFF3, as the parameter with the largest (AUC) of 0.9198 , showed the strongest prediction of a fall in GFR. However, limitation in the number of participants and variability of CAKUT categories leads to caution in interpretation of these results regarding the relationship of biomarkers to kidney scarring. Previously, in primary VUR, uNGAL was estimated as a predictive parameter of kidney scarring, in a large group of children but without correlation with GFR [10].

It has been shown in CAKUT that inflammatory molecules in urine are already increased in fetuses with PUV at a mean gestational age of 22 weeks, suggesting an early pathophysiological response of kidneys and urinary tract during embryonic formation of the urethral membrane [9]. Besides intense inflammation, fibrogenic mediator TGF- $\beta$ was also upregulated in urine in utero [9].
Recently, plasma, but not urinary NGAL, and urinary $\mathrm{FABP} /$ creatinine were proposed as potential markers of tubular injury in children with obstructive uropathy [3]. Anand et al. also revealed uNGAL as a useful biomarker, which correlates with plasma NGAL in children with congenital uropathy [2]. Another study also highlighted urinary NGAL in pediatric urinary obstruction [11], where a positive correlation between UNGAL and loss of kidney function detected by renal scintigraphy was observed [11], as confirmed by the recent study [2]. The strength of the cited results can be seen in the correction of measured urinary biomarkers to creatinine $(\mathrm{Cr})$, yet these results need to be validated in larger studies. It was noted that after pyeloplasty, uNGAL levels could stay elevated on follow-up, suggesting this is a marker of ongoing tubular injury besides relief of obstruction [3]. Moreover, a recent whole-genome expression study revealed elevated levels of expression of $L C N 2$ gene in CAKUT ureter tissue, removed by pyeloplasty, indicating that not only epithelial tubular cells are injured, but also epithelial cells from ureters [12], and furthermore that uNGAL could be originating from kidneys as well as from ureters.

However, present discrepancies among results related to repeatedly estimated biomarkers for progressive kidney damage in children with CAKUT, and wider, should be overcome by larger studies optimized to address all challenges mentioned in the previous paragraphs. Only then can we proceed along the kidney damage biomarker development roadmap.

Besides proteome analysis of urine, another promising source of biomarkers for kidney disease is urinary extracellular vesicles (uEV) (including exosomes). Although whole urine contains uEVs, the uEVs have a set of unique proteins compared with whole urine. Isolation of uEVs increases the identification rate of low-abundance proteins that are likely derived from kidney epithelial cells and could not be identified in whole urine, probably because they are masked by more abundant, plasma-derived proteins. uEVs could offer an even wider panel of putative biomarker molecules beside proteins. They contain mRNA and microRNA (miRNA) - the small noncoding RNAs that regulate mRNA processing-which provide the molecular signature similar to cells from kidney, bladder, or ureter tissue that release EVs. So, analysis of uEVs provides advantages that are warranted by biopsy of the target tissue and, thus, are designated as noninvasive "liquid biopsy." Moreover, analysis of posttranslational modification of plasma membrane proteins in uEVs may be another method for biomarker discovery with high protein specificity for a particular phenotype, as recently determined in autosomal dominant polycystic kidney disease (ADPKD) [13]. Furthermore, the EVs may constitute a therapeutic carrier that can contribute to attenuation of kidney fibrosis and repair of kidney injury [14]. 


\section{TFF3 as a new marker of CKD in CAKUT}

Many studies have suggested that TFF3 peptide performs important functions, such as wound healing, mucosal protection, cell proliferation, and cell migration in vivo and in vitro. It is a secretory product of mucin-producing epithelial cells, which are predominantly localized in the proximal and distal tubules and collecting duct, basal and intermediate layers of the urothelium, ureter and urethra [15]. Possible triggers for the release of TFF3 may include damage or inflammation, and until 2011 [16], urinary levels of TFF3 were associated with acute kidney injury only in animal models [17]. Elevation of TFF3 was proposed to be the cause of restitution of kidney function in different ways, according to results of in vitro studies [18].

TFF3, both the mRNA and the peptide, were recognized as a biomarker candidate, which needed validation. In a large prospective randomized study of more than 1000 participants with different diseases, the association of serum and urine TFF3 and severity of CKD was determined and its expression was confirmed in tubular epithelial cells [19]. Recently, the first study that deals with urinary TFFs in children with CAKUT observed the predictive role of TFF3 in progressive deterioration of kidney function [2]. In a previous study, elevation of urinary TFF1 was detected in lower CKD stages and TFF3 excretion was increased in CKD stages 4 and 5 [20]. The strength of the recent study is in measuring both NGAL, as a known marker of tubular injury, and TFF3 as a new proposed marker, in order to elucidate an independent association of the latter with progressive kidney loss of function [2]. TFF3 is a multifunctional factor with roles in regeneration and restitution of kidney damage $[15,16]$, which shows properties to link inflammation with tissue remodeling processes. Epithelial cells can undergo epithelial-mesenchymal transition (EMT) and in kidney fibrosis, interstitial fibroblasts are responsive to excessive matrix deposition that occurs in tubular epithelium close to loci with intense inflammation [21].

It was hypothesized that TFF3 participates in EMT, along with, e.g., TGF-beta and matrix metalloproteinases (MMPs), previously known to be important in kidney homeostasis and diseases. The link between TFF3 and MMPs was recently shown by increased production of MMPs (MMPs 1, 3, and 13) induced by recombinant TFF3 protein, in vitro [22]. However, gene variants of MMPs 1, 3, and 8, shown to result in dysregulation of MMP production, have been associated with CAKUT in children with obstructive uropathies [23]. Accordingly, the investigation of urinary TFF3 and MMPs might help in gaining a better understanding of the role of TFF3 in kidney damage and long-term CKD. Data regarding the regulation of TFF3 signaling pathways that mediate the effects of TFFs and even the TFF peptide receptor have not been fully comprehended yet. Recently, research on Tff3deficient mice pointed out Tff3-related biological pathways that might be regulated by a miRNA regulatory network [24]. Surprisingly, one of the 21 significantly deregulated miRNAs in Tff3 KO mice was upregulated miR-144, which is also upregulated in ureters from CAKUT patients [25]. Since miRNAs represent one class of important regulatory molecules involved in modulating gene expression at the post-transcriptional level, these results could trace a new avenue of research on regulation of TFF3 expression in progression of loss of kidney function, especially in CAKUT patients.

As many players are involved in CKD development and progression, it is of interest to perceive the most potent therapeutic targets. Currently, standard therapy, angiotensin Iconverting enzyme inhibitors (ACEI) or angiotensin receptor blockers (ARBs) can target hypertension (HT) and proteinuria and ameliorate fibrosis, but cannot completely suppress kidney damage progression. HT contributes to more rapid functional deterioration of kidneys in children secondary to CAKUT and renal dysplasia [26]. Decline in the annual glomerular filtration rate (GFR) is higher in HT children with CKD stage 2-4 of non-glomerular origin (CAKUT) than in normotensive ones [27]. Nevertheless, CAKUT children have predominantly tubular dysfunction and are at lower risk for HT than children with glomerulopathies. The ItalKid registry study showed no clear evidence that the use of ACEI slowed the progression of CKD in children with hypoplastic nephropathy [28]. However, ACE which is targeted by ACEI is regulated by well-known ACE I/D gene polymorphism and DD genotype has been associated with higher expression of ACE and synthesis of Ang II, an inflammatory and profibrotic factor, targeted by ARB, which could be involved in kidney scarring [29]. It would be of value to add data about HT, proteinuria and kidney scarring, and even genetics, in future biomarker studies of CKD in CAKUT.

\section{Behind the main scene and future perspectives}

Elucidation of genetic, epigenetic, environmental background, and molecular functions of putative biomarkers is still essential for understanding of both CAKUT and CKD. Animal model studies are of great importance in such research. Mouse models have been mostly used for functional research and validation of genetic variations identified in human studies of CAKUT. Two recent studies established causality/functionality of genes during mammalian kidney development by addressing the missense of genes [30] or gene expression regulation by alternative splicing that affects mesenchymal-to-epithelial transformation [31] and open future perspectives of kidney function/ dysfunction research. Tubulointerstitial fibrosis and extracellular matrix accumulation in the kidney interstitium during fibrosis, could be up- and down-regulated and even reversed by drug targeting in animal models. Also, zebrafish (Danio rerio) 
is a valuable model for large-scale, high-throughput screening studies for kidney organogenesis and functional validation of putative biomarkers for kidney deterioration/regeneration in different environmental/epigenetic settings of CAKUT that could be transferred to humans. Furthermore, novel widely used machine-learning methodology will be applied to CAKUT phenotyping toward modelling of common genetic kidney disease and elucidation of disease-causing genes. Research assumes development of a novel animal model for cystic kidney diseases in Xenopus tropicalis designed using gene editing, and the project (https://cordis.europa.eu/project/ $\mathrm{id} / 891127 / \mathrm{de}$ ) starts in the year in which the Nobel prize acknowledged the CRISPR/Cas9 methodology. Modelling of kidney organoids is another promising approach for validation of both genetic and environmental contributors to CAKUT and consequent CKD. Extended research into TFF3 as a new urinary biomarker of CKD in CAKUT [2] might be possible in future, in advanced versions of kidney organoids, with defects of the outflow tract where it may be possible to model urinary filtrate secretion and monitoring with a pool of urinary markers of kidney injury. In addition, drug screening towards modification of the target cell phenotype, which leads to protection of kidney function is envisaged through development of more complex organoids.

\section{Conclusion}

In conclusion, in the light of promising results, future research into biomarkers of CKD in CAKUT should be intensified harboring the multifactorial models of predictions and should include replicate studies. These efforts should strengthen national registries to enable follow-up and overcome boundaries toward validation and application of clinical biomarkers.

Funding The work was supported by the Serbian Ministry of Education, Science and Technological Development

\section{References}

1. Kinsner-Ovaskainen A, Morris J, Garne E, Loane M, Lanzoni M (2020) European Monitoring of Congenital Anomalies: JRCEUROCAT Report on Statistical Monitoring of Congenital Anomalies (2008 - 2017), EUR 30158 EN, Publications Office of the European Union, Luxembourg. https://doi.org/10.2760/575186

2. Anand S, Bajpai M, Khanna T, Kumar A (2020) Urinary biomarkers as point-of-care tests for predicting progressive deterioration of kidney function in congenital anomalies of kidney and urinary tract: Trefoil family factors (TFFs) as the emerging biomarkers. Pediatr Nephrol. https://doi.org/10.1007/s00467-02004841-8

3. McLeod DJ, Sebastião YV, Ching CB, Greenberg JH, Furth SL, Becknell B (2020) Longitudinal kidney injury biomarker trajectories in children with obstructive uropathy. Pediatr Nephrol 35: 1907-1914
4. Harambat J, van Stralen KJ, Kim JJ, Tizard EJ (2012) Epidemiology of chronic kidney disease in children. Pediatr Nephrol 27:363-373

5. Hogg RJ, Furth S, Lemley KV, Portman R, Schwartz GJ, Coresh J, Balk E, Lau J, Levin A, Kausz AT, Eknoyan G, Levey AS, National Kidney Foundation's Kidney Disease Outcomes Quality Initiative (2003) National Kidney Foundation's Kidney Disease Outcomes Quality Initiative clinical practice guidelines for chronic kidney disease in children and adolescents: evaluation, classification, and stratification. Pediatrics 111:1416-1421

6. Schwartz GJ, Muñoz A, Schneider MF, Mak RH, Kaskel F, Warady BA, Furth SL (2009) New equations to estimate GFR in children with CKD. J Am Soc Nephrol 20:629-637

7. Hsu CY, Ballard S, Batlle D, Bonventre JV, Böttinger EP, Feldman HI, Klein JB, Coresh J, Eckfeldt JH, Inker LA, Kimmel PL, Kusek JW, Liu KD, Mauer M, Mifflin TE, Molitch ME, Nelsestuen GL, Rebholz CM, Rovin BH, Sabbisetti VS, Van Eyk JE, Vasan RS, Waikar SS, Whitehead KM, Nelson RG, CKD Biomarkers Consortium (2015) Cross-disciplinary biomarkers research: lessons learned by the CKD Biomarkers Consortium. Clin J Am Soc Nephrol 10:894-902

8. Bartoli F, Pastore V, Calè I, Aceto G, Campanella V, Lasalandra C, Magaldi S, Niglio F, Basile A, Cocomazzi R (2019) Prospective study on several urinary biomarkers as indicators of renal damage in children with CAKUT. Eur J Pediatr Surg 29:215-222

9. Vieira ÉLM, Pessoa Rocha N, Macedo Bastos F, da Silveira KD, Pereira AK, Araújo Oliveira E, Marques de Miranda DM, Simões E, Silva AC (2017) Posterior urethral valve in fetuses: evidence for the role of inflammatory molecules. Pediatr Nephrol 32:1391-1400

10. Parmaksiz G, Noyan A, Dursun H, İnce E, Anarat R, Cengiz N (2016) Role of new biomarkers for predicting renal scarring in vesicoureteral reflux: NGAL, KIM-1, and L-FABP. Pediatr Nephrol 31:97-103

11. Bieniaś B, Sikora P (2018) Potential novel biomarkers of obstructive nephropathy in children with hydronephrosis. Dis Markers 2018:1015726

12. Jovanovic I, Zivkovic M, Kostic M, Krstic Z, Djuric T, Licastro D, Meroni G, Alavantic D, Stankovic A (2018) Transcriptome-driven integrative exploration of functional state of ureter tissue affected by CAKUT. Life Sci 212:1-8

13. Salih M, Demmers JA, Bezstarosti K, Leonhard WN, Losekoot M, van Kooten C, Gansevoort RT, DJM P, Zietse R, Hoorn EJ, DIPAK Consortium (2016) Proteomics of urinary vesicles links plakins and complement to polycystic kidney disease. J Am Soc Nephrol 27: 3079-3092

14. Shi Z, Wang Q, Zhang Y, Jiang D (2020) Extracellular vesicles produced by bone marrow mesenchymal stem cells attenuate renal fibrosis, in part by inhibiting the RhoA/ROCK pathway, in a UUO rat model. Stem Cell Res Ther 11:253

15. Rinnert M, Hinz M, Buhtz P, Reiher F, Lessel W, Hoffmann W (2010) Synthesis and localization of trefoil factor family (TFF) peptides in the human urinary tract and TFF2 excretion into the urine. Cell Tissue Res 339:639-647

16. Astor BC, Köttgen A, Hwang SJ, Bhavsar N, Fox CS, Coresh J (2011) Trefoil factor 3 predicts incident chronic kidney disease: a case-control study nested within the Atherosclerosis Risk in Communities (ARIC) study. Am J Nephrol 34:291-297

17. Taupin D, Podolsky DK (2003) Trefoil factors: initiators of mucosal healing. Nat Rev Mol Cell Biol 4:721-732

18. Zwaini Z, Alammari D, Byrne S, Stover C (2016) Mode of Proximal Tubule Damage: Differential Cause for the Release of TFF3? Front Immunol 7:122

19. Du TY, Luo HM, Qin HC, Wang F, Wang Q, Xiang Y, Zhang Y (2013) Circulating serum trefoil factor 3 (TFF3) is dramatically increased in chronic kidney disease. PLoS One 8:e80271 
20. Lebherz-Eichinger D, Tudor B, Ankersmit HJ, Reiter T, Haas M, Roth-Walter F, Krenn CG, Roth GA (2015) Trefoil Factor 1 Excretion is increased in early stages of chronic kidney disease. PLoS One 10:e0138312

21. Lovisa S, LeBleu VS, Tampe B, Sugimoto H, Vadnagara K, Carstens JL, Wu CC, Hagos Y, Burckhardt BC, PentchevaHoang T, Nischal H, Allison JP, Zeisberg M, Kalluri R (2015) Epithelial-to-mesenchymal transition induces cell cycle arrest and parenchymal damage in renal fibrosis. Nat Med 21:998-1009

22. Rösler S, Haase T, Claassen H, Schulze U, Schicht M, Riemann D, Brandt J, Wohlrab D, Müller-Hilke B, Goldring MB, Sel S, Varoga D, Garreis F, Paulsen FP (2010) Trefoil factor 3 is induced during degenerative and inflammatory joint disease, activates matrix metalloproteinases, and enhances apoptosis of articular cartilage chondrocytes. Arthritis Rheum 62:815-825

23. Djuric T, Zivkovic M, Milosevic B, Andjelevski M, Cvetkovic M, Kostic M, Stankovic A (2014) MMP-1 and -3 haplotype is associated with congenital anomalies of the kidney and urinary tract. Pediatr Nephrol 29:879-884

24. Shah AA, Leidinger P, Keller A, Wendschlag A, Backes C, BausLoncar M, Meese E, Blin N (2011) The intestinal factor Tff3 and a miRNA network regulate murine caloric metabolism. RNA Biol 8: $77-81$

25. Jovanovic I, Zivkovic M, Kostic M, Krstic Z, Djuric T, Kolic I, Alavantic D, Stankovic A (2016) Transcriptome-wide based identification of miRs in congenital anomalies of the kidney and urinary tract (CAKUT) in children: the significant upregulation of tissue miR-144 expression. J Transl Med 14:193

26. Gonzalez Caledon C, Bitsori M, Tullus K (2007) Progression of chronic renal failure in children with dysplastic kidneys. Pediatr Nephrol 22:1014-1020
27. Fathallah-Shaykh SA, Flynn JT, Pierce CB, Abraham AG, BlydtHansen TD, Massengill SF, Moxey-Mims MM, Warady BA, Furth SL, Wong CS (2015) Progression of pediatric CKD of nonglomerular origin in the CKiD cohort. Clin J Am Soc Nephrol 10:571-577

28. Ardissino G, Viganò S, Testa S, Daccò V, Paglialonga F, Leoni A, Belingheri M, Avolio L, Ciofani A, Claris-Appiani A, Cusi D, Edefonti A, Ammenti A, Cecconi M, Fede C, Ghio L, La Manna A, Maringhini S, Papalia T, Pela I, Pisanello L, Ratsch IM, ItalKid Project (2007) No clear evidence of ACEi efficacy on the progression of chronic kidney disease in children with hypodysplastic nephropathy-report from the ItalKid Project database. Nephrol Dial Transplant 22:2525-2530

29. Kostić M, Stanković A, Zivković M, Peco-Antić A, Jovanović O, Alavantić D, Kruscić D (2004) ACE and AT1 receptor gene polymorphisms and renal scarring in urinary bladder dysfunction. Pediatr Nephrol 19:853-857

30. Zhang C, Balbo B, Ma M, Zhao J, Tian X, Kluger Y, Somlo S (2020) Cyclin-Dependent Kinase 1 Activity Is a Driver of Cyst Growth in Polycystic Kidney Disease. J Am Soc Nephrol. https:// doi.org/10.1681/ASN.2020040511

31. Wineberg Y, Bar-Lev TH, Futorian A, Ben-Haim N, Armon L, Ickowicz D, Oriel S, Bucris E, Yehuda Y, Pode-Shakked N, Gilad S, Benjamin S, Hohenstein P, Dekel B, Urbach A, Kalisky T (2020) Single-Cell RNA Sequencing Reveals mRNA Splice Isoform Switching during Kidney Development. J Am Soc Nephrol 31:2278-2291

Publisher's note Springer Nature remains neutral with regard to jurisdictional claims in published maps and institutional affiliations. 